\title{
ON OPTIMALITY OF BOLD PLAY FOR DISCOUNTED DUBINS-SAVAGE GAMBLING PROBLEMS WITH LIMITED PLAYING TIMES
}

\author{
YI-CHING YAO, ${ }^{*}$ Academia Sinica and National Chengchi University
}

\begin{abstract}
In the classic Dubins-Savage subfair primitive casino gambling problem, the gambler can stake any amount in his possession, winning $(1-r) / r$ times the stake with probability $w$ and losing the stake with probability $1-w, 0 \leq w \leq r \leq 1$. The gambler seeks to maximize the probability of reaching a fixed fortune by gambling repeatedly with suitably chosen stakes. This problem has been extended in several directions to account for limited playing time or future discounting. We propose a unifying framework that covers these extensions, and prove that bold play is optimal provided that $w \leq \frac{1}{2} \leq r$. We also show that this condition is in fact necessary for bold play to be optimal subject to the constraint of limited playing time.
\end{abstract}

Keywords: Gambling theory; primitive casino; red-and-black; roulette; discount factor; optimal strategy

2000 Mathematics Subject Classification: Primary 60G40

Secondary 91A60

\section{Introduction}

In their classic work, Dubins and Savage [10], [11] introduced the primitive casino gambling problem in which a gambler can stake any amount $s$ of his current fortune $f, 0 \leq s \leq f<1$. If he stakes an amount $s$, his fortune becomes $f+\bar{r} s / r$ with probability $w$ and $f-s$ with probability $\bar{w}=1-w$, where $0<r \leq 1$ and $0 \leq w \leq 1$ are fixed constants (and $\bar{r}=1-r$ ). While he is allowed to gamble repeatedly, the gambler's objective is to maximize the probability of reaching a fortune of 1 (the goal). In an ingenious proof, Dubins and Savage showed that it is optimal to play boldly if the primitive casino is subfair (i.e. $w \leq r$ ). Here the word 'subfair' is used in the weak sense, meaning 'fair or subfair', and to play boldly is to stake on each play as much as possible without risking overshooting the goal, i.e. to stake $\min \{f,(1-f) r / \bar{r}\}$ if the current fortune is $f, 0<f<1$. The Dubins-Savage optimality result on the bold strategy has been generalized in several directions, as summarized below. Let $f_{n}$ denote the fortune at the end of the $n$th play, $n=1,2, \ldots$, with $f_{0}=f$ being the initial fortune.

(i) Subfair primitive casinos with limited playing times. Under the requirement that the total number of plays be limited to a prescribed integer value $n>0$, Dvoretzky proved (see [11, pp. 92-95]) that the bold strategy remains optimal provided that $w \leq r=\frac{1}{2}$ (the subfair 'redand-black' casino setting) or that $\frac{1}{2}=w \leq r$ (the 'taxed-coin' casino setting). Dvoretzky also constructed examples demonstrating that the bold strategy is not optimal in general for subfair

Received 14 July 2006; revision received 28 October 2006.

* Postal address: Institute of Statistical Science, Academia Sinica, Taipei, 115, Taiwan, R. O. C.

Email address: yao@stat.sinica.edu.tw

Supported in part by a grant from the National Science Council of Taiwan. 
primitive casinos with limited playing times [11, pp. 110-111]. Beyond those for subfair redand-black and taxed-coin casinos, no further optimality results on the bold strategy have been published in the literature. The present study was motivated by this, and our main results show that for each $n \geq 3$ the condition that $w \leq \frac{1}{2} \leq r$ is necessary and sufficient for the bold strategy to be optimal for all initial fortunes $f \in(0,1)$ when the gambler is allowed to play at most $n$ times.

(ii) Discounted, subfair primitive casinos. The gambler seeks to maximize the expected utility earned upon reaching the fortune 1 (the goal), where the utility at the goal is $\beta^{n}$ (and $\beta$, $0 \leq \beta \leq 1$, is the discount factor) if the goal is reached on the $n$th play. In other words, it is desired to find a strategy that maximizes $\mathrm{E} \beta^{N} \mathbf{1}_{\{N<\infty\}}$, where $N=\inf \left\{n: f_{n} \geq 1\right\}$ (the number of plays needed to reach the goal) and $\mathbf{1}_{A}$ denotes the indicator of the (generic) set $A$. Note that this discounted version of the Dubins-Savage gambling problem is equivalent to the following formulation; see [14]. If the gambler stakes an amount $s$ of his fortune $f$, then his fortune becomes $f+\bar{r} s / r, f-s$, or 0 with respective probability $\beta w, \beta \bar{w}$, or $\bar{\beta}=1-\beta$. The objective remains to maximize the probability of reaching the fortune 1 . For discounted, subfair red-and-black casinos ( $w \leq r=\frac{1}{2}$ ), Klugman [14] proved that the bold strategy is optimal for all $\beta$. This result was extended to discounted, subfair primitive casinos by Chen and Zame [5], who showed that the bold strategy is optimal provided that $w \leq \frac{1}{2} \leq r$ or that $\frac{1}{2} \leq w \leq r \geq(\sqrt{5}-1) / 2$. On the other hand, Chen [3] found for discounted, subfair primitive casinos that if either $0<w \leq r<\frac{1}{2}$ or $\frac{1}{2}<w \leq r<(\sqrt{5}-1) / 2$, then there exists an initial fortune $f \in(0,1)$ for which the bold strategy is not optimal for sufficiently small $\beta>0$.

(iii) Subfair primitive casinos in the presence of inflation. While the objective remains to maximize the probability of reaching the (discounted) fortune 1, it is assumed that the gambler's fortune is discounted by a factor of $\alpha, 0<\alpha \leq 1$, after each play. (Note that $\bar{\alpha} / \alpha=(1-\alpha) / \alpha \geq 0$ may be interpreted as the inflation rate.) Then if the gambler stakes an amount $s$ of his fortune $f$, $0 \leq s \leq f$, his (discounted) fortune becomes $\alpha(f+\bar{r} s / r)$ with probability $w$ and $\alpha(f-s)$ with probability $\bar{w}$. Chen [4] proved the optimality of the bold strategy for subfair red-and-black casinos with inflation, and this was recently extended to subfair primitive casinos with inflation by Chen et al. [7], who showed that the bold strategy is optimal provided that $w \leq \frac{1}{2} \leq r$. On the other hand, Chen et al. [6] found that the bold strategy is no longer optimal if $0<w \leq r<\frac{1}{2}$ and the inflation rate, $\bar{\alpha} / \alpha$, is between $r / \bar{r}$ and $\bar{r} / r$.

(iv) Subfair roulette casinos. In a subfair roulette casino governed by two parameters $w$ and $r, 0 \leq w \leq r \leq 1$, if the gambler stakes amounts $s_{1}, s_{2}, \ldots, s_{k}$ of his fortune $f<1$ on $k$ different numbers (with $k w \leq 1$ and $S:=\sum_{j=1}^{k} s_{j} \leq f$ ), then his fortune becomes $f-S+s_{i} / r, i=1, \ldots, k$, with probability $w$ and $f-S$ with probability $1-k w$. Note that this reduces to the primitive casino setting if the gambler is allowed to bet on only one number in each play. (In many real-world casinos, $w=\frac{1}{38}$ and $r=\frac{1}{36}$.) It was proved by Smith [22] and Dubins [8] that the maximum probability of reaching the fortune 1 is attained by the bold strategy that stakes, on each play, an amount $\min \{f,(1-f) r / \bar{r}\}$ of the current fortune $f$ on only one number.

(v) Subfair red-and-black casinos with house limits. When there is a house limit $z, 0<z<\frac{1}{2}$, the bold strategy is to stake an amount $\min \{f, 1-f, z\}$ of the fortune $f$. Wilkins [24] established the optimality of the bold strategy for subfair red-and-black casinos with a house limit $z=1 / n$, for any integer $n \geq 3$. This result was extended by Chen [2] to discounted subfair red-andblack casinos. Heath et al. [13] showed, however, that if the house limit $z$ either satisfies 
$1 /(n+1)<z<1 / n$ for some integer $n \geq 3$ or is irrational and satisfies $\frac{1}{3}<z<\frac{1}{2}$, then there exists an $\varepsilon>0$ such that for $0<w<\varepsilon$ the bold strategy is not optimal for some initial fortune $f$. Furthermore, with $B_{w, z} \subset(0,1)$ denoting the set of initial fortunes for which the bold strategy is optimal, Heath et al. [13] showed that if $1 /(n+1)<z<1 / n$ for some integer $n \geq 3$, then $B_{w, z}$ has Lebesgue measure 0 for some $w<\frac{1}{2}$. The former result of Heath $e t$ al. [13] was recently improved by Schweinsberg [20], who showed that, for all irrational $z<\frac{1}{2}$ and $0<w<\frac{1}{2}$, the bold strategy is not optimal for some initial fortune $f$.

(vi) Subfair fortune-dependent red-and-black casinos. In this case, if the gambler stakes an amount $s$ of his fortune $f$, then his fortune becomes $f+s$ with probability $w \equiv w(f)$ and $f-s$ with probability $1-w(f)$, where the win probability $w$ depends on the gambler's fortune $f$. Dubins [9] constructed an example to show that the bold strategy is not necessarily optimal even when $w(f)<\frac{1}{2}$ for all $f$. It will be of theoretical interest to find general conditions on $w(f)$ under which the bold strategy is optimal.

(vii) Continuous-time gambling problems. Motivated by Dubins-Savage discrete-time gambling theory, Heath and Sudderth [12], Pestien and Sudderth [17], [18], and Sudderth and Weerasinghe [23] have introduced and solved some continuous-time gambling problems in which a player (gambler) starts at $x \in(0,1)$ and seeks to maximize the probability of reaching 1 . The process, $\{X(t): t \geq 0\}$, of the player's positions (fortunes) is a diffusion process whose drift and diffusion parameters are chosen by the player at each instant of time from a set depending on the current position. Of particular interest is a result in [23] showing that the bold strategy is optimal for continuous-time subfair red-and-black and roulette casinos with limited playing times. In contrast to the discrete-time setting, where no explicit expression is available for the optimal value function in general, the continuous-time theory often allows one to solve a problem by checking the conditions of a corresponding 'verification lemma' for the explicit value function of a guessed optimal strategy (see [18]).

While in the present study we consider only subfair casinos, for which bold play is of primary concern, there have been extensive studies of superfair casinos and various generalizations, for which timid play is of special interest; see, e.g. [1], [9], [15], [16], [17], [19], and [21].

In Section 2 we propose a unifying framework involving the parameters, $r, w, n$ (the number of plays allowed), and $\alpha$ and $\beta$ (which are discount factors), that cover cases (i)-(iv). It is shown that the bold strategy is optimal for all $n, \alpha$, and $\beta$ if $w \leq \frac{1}{2} \leq r$. In particular, the bold strategy is optimal for the original Dubins-Savage primitive casino gambling problem with limited playing time provided that $w \leq \frac{1}{2} \leq r$ (which includes the subfair red-and-black and taxedcoin casinos as special cases). In Section 3 it is shown that the condition $w \leq \frac{1}{2} \leq r$ is in fact necessary for the bold strategy to be optimal subject to the constraint of limited playing time. Section 4 contains the proof of the technical result Lemma 1.

\section{The unifying framework and optimality of the bold strategy}

We first consider the discounted primitive casino case. Under the unifying framework, if the gambler stakes an amount $s$ of his fortune $f$, then his fortune becomes $\alpha(f+\bar{r} s / r), \alpha(f-s)$, or 0 with respective probability $\beta w, \beta \bar{w}$, or $\bar{\beta}$. The objective is to maximize the probability of reaching fortune 1 in at most $n$ plays, $n=1,2, \ldots, \infty(n=\infty$ corresponding to the case of unlimited playing time). Note that cases (i), (ii), and (iii) in Section 1 correspond to the parameter choices $\alpha=\beta=1$ and $n<\infty ; \alpha=1,0<\beta \leq 1$, and $n=\infty$; and $0<\alpha \leq 1$, $\beta=1$, and $n=\infty$, respectively. 
Let $U_{0}(f)=\mathbf{1}_{[1, \infty)}(f), f \geq 0$. For $n=0,1, \ldots$, recursively define

$U_{n+1}(f)= \begin{cases}\sup \left\{\beta \bar{w} U_{n}(\alpha(f-y))+\beta w U_{n}(\alpha(f+y \bar{r} / r)): 0 \leq y \leq B(f)\right\}, & 0 \leq f<1, \\ 1, & f \geq 1,\end{cases}$

where the bold stake, $B(f)$, is defined as

$$
\begin{aligned}
B(f) & =\min \left\{f,\left(\alpha^{-1}-f\right) r / \bar{r}\right\}, \quad 0 \leq f<1, \\
& = \begin{cases}f, & 0 \leq f \leq r / \alpha, \\
\left(\alpha^{-1}-f\right) r / \bar{r}, & r / \alpha \leq f<1 .\end{cases}
\end{aligned}
$$

It is easily seen that $U_{n}(f)$ is the maximum probability of attaining fortune 1 (the goal) with initial fortune $f$ when the gambler is allowed to play at most $n$ times. Clearly, as $n$ tends to $\infty, U_{n}(f)$ increases to $U_{\infty}(f)$, the maximum probability of attaining the goal with initial fortune $f$ when there is unlimited playing time (see [11, Section 2.15] and [16, Section 3.6]). It will be shown for $w \leq \frac{1}{2} \leq r$ that the supremum in (1) is attained at $y=B(f)$, from which it follows that the bold strategy is optimal. To this end, define $Q_{0}(f)=\mathbf{1}_{[1, \infty)}(f), f \geq 0$, and, for $n=0,1, \ldots$,

$$
Q_{n+1}(f)= \begin{cases}\beta \bar{w} Q_{n}(\alpha(f-B(f)))+\beta w Q_{n}(\alpha(f+B(f) \bar{r} / r)), & 0 \leq f<1, \\ 1, & f \geq 1\end{cases}
$$

Then $Q_{n}(f)$ is the probability of attaining the goal in $n$ plays under the bold strategy (with initial fortune $f$ ). As $n$ tends to $\infty, Q_{n}(f)$ increases to $Q_{\infty}(f)$, the probability of attaining the goal when the gambler uses the bold strategy with unlimited playing time.

Theorem 1. Assume that $0 \leq w \leq \frac{1}{2} \leq r \leq 1$ and $0 \leq \alpha, \beta \leq 1$. Then $Q_{n}(f)=U_{n}(f)$ for $f \geq 0$ and $n=0,1, \ldots, \infty$.

Theorem 1 shows that the bold strategy is optimal provided that $w \leq \frac{1}{2} \leq r$, which includes some of the results of [4], [5], [7], [10], [11], and [14] as special cases.

Proof. The theorem is trivially true if $w=0, r=1, \alpha=0, \beta=0$, or $\alpha \leq r$. We now assume that $0<w \leq \frac{1}{2} \leq r<1,0<\alpha, \beta \leq 1$, and $\alpha>r$. It follows from (3) and Lemma 1 , below, that

$Q_{n+1}(f)= \begin{cases}\sup \left\{\beta \bar{w} Q_{n}(\alpha(f-y))+\beta w Q_{n}(\alpha(f+y \bar{r} / r)): 0 \leq y \leq B(f)\right\}, & 0 \leq f<1, \\ 1, & f \geq 1 .\end{cases}$

In view of (1), $Q_{n}$ and $U_{n}$ satisfy the same recursion equation with the same initial condition, $Q_{0}=U_{0}$. Thus, $Q_{n}(f)=U_{n}(f)$ for all finite $n$ and

$$
Q_{\infty}(f)=\lim _{n \rightarrow \infty} Q_{n}(f)=\lim _{n \rightarrow \infty} U_{n}(f)=U_{\infty}(f) .
$$

The proof is complete.

Lemma 1. Assume that $0<w \leq \frac{1}{2} \leq r<1,0<\alpha, \beta \leq 1$, and $\alpha>r$. Then, for $n=0,1, \ldots$, and $0 \leq y \leq f<1$,

$$
Q_{n+1}(f) \geq \beta \bar{w} Q_{n}(\alpha(f-y))+\beta w Q_{n}(\alpha(f+y \bar{r} / r)) .
$$


The proof of Lemma 1, which is long, is relegated to Section 4.

We next consider the case of a discounted roulette casino governed by the parameters $w, r$, $\alpha, \beta$, and $n$. Under the unifying framework, if the gambler stakes amounts $s_{1}, \ldots, s_{k}$ of his fortune $f$ on $k$ different numbers, then his fortune becomes $\alpha\left(f-S+s_{i} / r\right), i=1, \ldots, k$, with probability $\beta w, \alpha(f-S)$ with probability $\beta(1-k w)$, and 0 with probability $\bar{\beta}=1-\beta$, where $S:=s_{1}+\cdots+s_{k} \leq f$ and $k w \leq 1$. Note that case (iv) in Section 1 corresponds to the parameter choices $\alpha=\beta=1$ and $n=\infty$. Letting $U_{0}^{*}(f)=\mathbf{1}_{[1, \infty)}(f), f \geq 0$, for $n=0,1, \ldots$, we recursively define

$$
U_{n+1}^{*}(f)=\left\{\begin{array}{rr}
\sup \left\{\beta(1-k w) U_{n}^{*}(\alpha(f-Y))+\beta w \sum_{i=1}^{k} U_{n}^{*}\left(\alpha\left(f-Y+\frac{y_{i}}{r}\right)\right):\right. \\
\left.k w \leq 1, y_{i} \geq 0, Y:=\sum_{j=1}^{k} y_{j} \leq f\right\}, \\
1, & 0 \leq f<1, \\
& f \geq 1 .
\end{array}\right.
$$

Clearly, $U_{n}^{*}(f)$ is the maximum probability of attaining fortune 1 when the gambler with initial fortune $f$ is allowed to play at most $n$ times in the discounted roulette casino. Also, as $n$ tends to $\infty, U_{n}^{*}(f)$ increases to $U_{\infty}^{*}(f)$, the maximum probability of reaching fortune 1 when the gambler can play indefinitely. (Obviously $Q_{n}(f) \leq U_{n}(f) \leq U_{n}^{*}(f)$.) The following theorem shows that, to attain the maximum probability of reaching fortune 1 , the gambler should stake an amount $B(f)$ of his fortune $f$ on only one number in each play, provided that $w \leq \frac{1}{2} \leq r$.

Theorem 2. Assume that $0 \leq w \leq \frac{1}{2} \leq r \leq 1$ and $0 \leq \alpha, \beta \leq 1$. Then $U_{n}^{*}(f)=U_{n}(f)$ for $f \geq 0$ and $n=0,1, \ldots, \infty$.

Proof. It suffices to show that, for $n=0,1, \ldots$ and for any stakes $y_{1} \geq y_{2} \geq \cdots \geq y_{k}>0$ with $k \geq 2, k w \leq 1$, and $Y:=y_{1}+\cdots+y_{k} \leq f<1$, we have

$$
\begin{aligned}
& \beta(1-k w) U_{n}^{*}(\alpha(f-Y))+\beta w \sum_{i=1}^{k} U_{n}^{*}\left(\alpha\left(f-Y+\frac{y_{i}}{r}\right)\right) \\
& \quad \leq \beta(1-(k-1) w) U_{n}^{*}\left(\alpha\left(f-Y^{\prime}\right)\right)+\beta w \sum_{i=1}^{k-1} U_{n}^{*}\left(\alpha\left(f-Y^{\prime}+\frac{y_{i}^{\prime}}{r}\right)\right),
\end{aligned}
$$

where $y_{i}^{\prime}=y_{i}-y_{k}$ and $Y^{\prime}=\sum_{i=1}^{k-1} y_{i}^{\prime}=Y-k y_{k}$. That is, when the gambler is allowed to play at most $n+1$ times, initially staking $y_{1} \geq \cdots \geq y_{k}$ on $k$ different numbers is dominated by staking $y_{1}^{\prime} \geq \cdots \geq y_{k-1}^{\prime}$ on $k-1$ numbers. Since $U_{n}^{*}(x)$ is nondecreasing in $x$, (5) follows easily upon noting that $r \geq \frac{1}{2}$ and $k \geq 2$ together imply that $Y-y_{i} / r \geq Y^{\prime}-y_{i}^{\prime} / r, i=1, \ldots, k$.

Remark 1. The above proof takes advantage of the following simple observation. In the undiscounted case, in which $\alpha=\beta=1$, for $r>\frac{1}{2}$ a gambler who stakes the same amount on two (or more) numbers is guaranteed to lose money even if the bet is won. Likewise, a gambler who bets on multiple numbers can always do better by reducing all bets by the same amount.

\section{Necessity of the condition $w \leq \frac{1}{2} \leq r$ for optimality of the bold strategy}

In this section we focus on the undiscounted case, in which $\alpha=\beta=1$. By Theorem 1 , for subfair primitive casinos with limited playing times, the bold strategy is optimal provided that 
$w \leq \frac{1}{2} \leq r$. On the other hand, Dvoretzky showed (see [11, Section 6.6]) that the bold strategy is not optimal for some initial fortune $f$ if $w<r<\frac{1}{2}$ or $\frac{2}{3}<w<r<2^{-1 / 2}$. It is natural to ask whether the condition $w \leq \frac{1}{2} \leq r$ of Theorem 1 is necessary for the bold strategy to be optimal. The following result answers this question in the affirmative.

Theorem 3. Let $3 \leq n<\infty, 0<w \leq r<1$, and $\alpha=\beta=1$. Then

(i) $Q_{n}(f)=U_{n}(f)$ for all $f \geq 0$ if and only if $w \leq \frac{1}{2} \leq r$, and

(ii) $Q_{n}(f)=U_{n}^{*}(f)$ for all $f \geq 0$ if and only if $w \leq \frac{1}{2} \leq r$.

Theorem 3 follows immediately from Theorems 1 and 2 and Lemmas 2 and 3, below.

Lemma 2. For $3 \leq n<\infty, 0<w \leq r<\frac{1}{2}, \alpha=\beta=1$, and $f \in\left[r^{n-2}(1-\bar{r}(\bar{r}-r)), r^{n-2}\right)$, we have

$$
Q_{n}(f)=w^{n-1}(1+\bar{w})<w^{n-1}(1+2 \bar{w}) \leq U_{n}(f) .
$$

Proof. The following argument is easily adapted from [11, Section 6.6, Example 1], and is included here for completeness. Under the bold strategy, the gambler goes broke if any of his first $n-2$ plays is unsuccessful, whereas his fortune increases to $f / r^{n-2} \in[1-\bar{r}(\bar{r}-r), 1)$ after $n-2$ successful plays. (Note that $1-\bar{r}(\bar{r}-r)>r$ since $r>0$.) If the $(n-1)$ th play is successful, the gambler attains the goal; otherwise, his fortune reduces to

$$
\frac{f / r^{n-2}-r}{\bar{r}} \in[2 r, 1) .
$$

In the latter case, the gambler attains the goal when the $n$th play is successful, so

$$
Q_{n}(f)=w^{n-2}(w+\bar{w} w)=w^{n-1}(1+\bar{w}) .
$$

To show that $U_{n}(f) \geq w^{n-1}(1+2 \bar{w})$, consider the following strategy. Stake boldly in the first $n-3$ plays, resulting in a total fortune of $f / r^{n-3} \in[r-r \bar{r}(\bar{r}-r), r)$ if the first $n-3$ plays are all successful. In the $(n-2)$ th play stake just enough to reach the fortune $r+r \bar{r}$ if successful, and then stake boldly in the remaining two plays. If the $(n-2)$ th play is successful (and the fortune thus becomes $r+r \bar{r}$ ), then the gambler can reach the goal when at least one of the remaining two (bold) plays is successful. If the $(n-2)$ th play is unsuccessful then the fortune becomes

$$
f / r^{n-3}-\left[r+r \bar{r}-f / r^{n-3}\right] r / \bar{r}=\left[f / r^{n-3}-r^{2}(1+\bar{r})\right] / \bar{r} \geq r^{2},
$$

in which case the gambler can still attain the goal when the remaining two (bold) plays are both successful. Hence, under this strategy, the gambler attains the goal with probability

$$
w^{n-3}\left[w(w+\bar{w} w)+\bar{w} w^{2}\right]=w^{n-1}(1+2 \bar{w}),
$$

completing the proof.

Lemma 3. For $3 \leq n<\infty, \frac{1}{2}<w \leq r<1, \alpha=\beta=1$, and

$$
f \in\left[r^{n-1}(1+2 \bar{r}), r^{n-2}(1+r \bar{r})\right),
$$

we have

$$
Q_{n}(f)=w^{n-2}<w^{n-1}(1+2 \bar{w}) \leq U_{n}(f)
$$


Proof. Under the bold strategy, the gambler goes broke if any of the first $n-3$ plays is unsuccessful, whereas his fortune becomes $f / r^{n-3} \geq r^{2}(1+2 \bar{r})>r$ after $n-3$ successful plays. If the $(n-2)$ th play is successful, then he attains the goal; otherwise, the fortune becomes

$$
\frac{f / r^{n-3}-r}{\bar{r}}<\frac{r+\bar{r} r^{2}-r}{\bar{r}}=r^{2} .
$$

In the latter case, the goal cannot be attained even if the remaining two plays are successful. Thus, $Q_{n}(f)=w^{n-2}$.

To show that $U_{n}(f) \geq w^{n-1}(1+2 \bar{w})$, consider the following strategy. Stake boldly in the first $n-3$ plays, so that the gambler's fortune becomes $f / r^{n-3}>r$ after $n-3$ successful plays (and he goes broke if any of the first $n-3$ plays is unsuccessful). In the $(n-2)$ th play stake $s=f / r^{n-3}-r^{2}>0$, and stake boldly in the remaining two plays. If the $(n-2)$ th play is unsuccessful, then the fortune reduces to $f / r^{n-3}-s=r^{2}$, which is just enough for the gambler to attain the goal with two successful bold plays. If the $(n-2)$ th play is successful, then the fortune increases to

$$
f / r^{n-3}+s \bar{r} / r=f / r^{n-2}-r \bar{r} \in[r+r \bar{r}, 1),
$$

meaning that the gambler can attain the goal when at least one of the remaining two bold plays is successful. Thus, this strategy enables the gambler to attain the goal with probability

$$
w^{n-3}\left[\bar{w} w^{2}+w(w+\bar{w} w)\right]=w^{n-1}(1+2 \bar{w}),
$$

completing the proof.

Remark 2. For $n=1,2$, it is readily seen that $Q_{n}(f)=U_{n}(f)$ for all $f \geq 0$ and all $w, r, \alpha$, and $\beta$.

Remark 3. For general values of $\alpha$ and $\beta, 0<\alpha, \beta \leq 1$, it seems much more complicated to determine the conditions on $w, r, \alpha$, and $\beta$ under which $Q_{n}(f)=U_{n}(f)$ for all $f \geq 0$. Note that the following generalization of Lemma 2 can be easily proved. For $3 \leq n<\infty$, $0<w \leq r<\frac{1}{2}, r<\alpha \leq 1,0<\beta \leq 1$, and $(r / \alpha)^{n-1}(1+2 \bar{r} / \alpha) \leq f<(r / \alpha)^{n-\overline{2}}$, we have

$$
Q_{n}(f)=(\beta w)^{n-1}(1+\beta \bar{w})<(\beta w)^{n-1}(1+2 \beta \bar{w}) \leq U_{n}(f),
$$

provided that $(r / \alpha)(1+2 \bar{r} / \alpha)<1$. However, it can be shown that if $0<w \leq r<\frac{1}{2}$ and $(r / \alpha)(1+2 \bar{r} / \alpha) \geq 1$, then $Q_{3}(f)=U_{3}(f)$ for all $f \geq 0$.

\section{Proof of Lemma 1}

Our proof of Lemma 1 is long and based on induction, and is similar in spirit to, but much more involved than, Dvoretzky's proof of the optimality of the bold strategy for subfair redand-black casinos with limited playing times (see [11, pp. 92-94]), while the detailed analysis in our proof is similar to that of [7, Theorem 1]. (In contrast to our, induction-based, proof, the latter proof makes use of [11, Theorem 2.12.1] by verifying that the value function of the bold strategy is excessive.) We will need the following two simple lemmas, which are essentially [7, Lemmas 1 and 2] and can be verified in a straightforward manner.

Lemma 4. Suppose that $f$ and $y$ satisfy $0<y<f<1$ and

$$
\frac{(f-y) \alpha^{2}}{r} \geq \frac{\alpha(f+y \bar{r} / r)-r / \alpha}{\bar{r} / \alpha} \geq 0 .
$$


Define

$$
\begin{aligned}
& f^{\prime} \equiv f^{\prime}(f, y)=2 \alpha f-\alpha(1-\bar{r} / r) y-r / \alpha, \\
& y^{\prime} \equiv y^{\prime}(f, y)=\{r / \alpha-\alpha(1-\bar{r} / r) f-2 y \alpha \bar{r} / r\} r / \bar{r} .
\end{aligned}
$$

Then

$$
\alpha\left(f^{\prime}+y^{\prime} \frac{\bar{r}}{r}\right)=(f-y) \frac{\alpha^{2}}{r} \geq \alpha\left(f^{\prime}-y^{\prime}\right)=\frac{\alpha(f+y \bar{r} / r)-r / \alpha}{\bar{r} / \alpha} \geq 0
$$

and $0 \leq y^{\prime} \leq f^{\prime}$.

Lemma 5. Suppose that $f$ and $y$ satisfy $0<y<f<1$ and

$$
\frac{\alpha(f+y \bar{r} / r)-r / \alpha}{\bar{r} / \alpha} \geq(f-y) \frac{\alpha^{2}}{r} \geq 0 .
$$

Define

$$
\begin{aligned}
f^{\prime} & \equiv f^{\prime}(f, y)=\frac{\left(\bar{r}^{2} / r+r\right) f+\left(\bar{r}-\bar{r}^{2} / r\right) y-r^{2} / \alpha^{2}}{\bar{r} / \alpha}, \\
y^{\prime} & \equiv y^{\prime}(f, y)=\frac{(r-\bar{r}) f+2 \bar{r} y-r^{2} / \alpha^{2}}{\bar{r} / \alpha} .
\end{aligned}
$$

Then

$$
\alpha\left(f^{\prime}+y^{\prime} \frac{\bar{r}}{r}\right)=\frac{\alpha(f+y \bar{r} / r)-r / \alpha}{\bar{r} / \alpha} \geq \alpha\left(f^{\prime}-y^{\prime}\right)=(f-y) \frac{\alpha^{2}}{r} \geq 0
$$

and $0 \leq y^{\prime} \leq f^{\prime}$.

Proof of Lemma 1. Note that $Q_{n}(f)$ is nondecreasing in $f$ and $n$, that

$$
\left.Q_{n+1}(f)=\beta w Q_{n}(f \alpha / r) \quad \text { if } B(f)=f \text { (i.e. } f \leq r / \alpha\right),
$$

and that

$$
Q_{n+1}(f)=\beta \bar{w} Q_{n}\left(\frac{f-r / \alpha}{\bar{r} / \alpha}\right)+\beta w \quad \text { if } B(f)=\left(\alpha^{-1}-f\right) \frac{r}{\bar{r}} \leq f\left(\text { i.e. } \frac{r}{\alpha} \leq f<1\right) .
$$

We proceed by induction on $n$. Clearly, (4) holds for $n=0$. Suppose that (4) holds for all $n<m, m \geq 1$. We now prove that, for $0 \leq y \leq f<1$,

$$
Q_{m+1}(f) \geq \beta \bar{w} Q_{m}(\alpha(f-y))+\beta w Q_{m}(\alpha(f+y \bar{r} / r)) .
$$

For $0 \leq y \leq(\bar{\alpha} / \alpha)(r / \bar{r}) f$, we have

$$
\alpha(f-y) \leq \alpha(f+y \bar{r} / r) \leq f
$$

whence

$$
Q_{m+1}(f) \geq Q_{m}(f) \geq \beta \bar{w} Q_{m}(\alpha(f-y))+\beta w Q_{m}(\alpha(f+y \bar{r} / r)) .
$$

(Note that the more intuitive reason for the above inequality to hold is that when $y$ is this small, the gambler's fortune decreases even if the bet is won.) For $B(f)<y \leq f$ (implying that $B(f)=\left(\alpha^{-1}-f\right) r / \bar{r}$ and $\left.f>r / \alpha\right)$, by (7) we have

$$
\begin{aligned}
Q_{m+1}(f) & =\beta \bar{w} Q_{m}(\alpha(f-B(f)))+\beta w \\
& \geq \beta \bar{w} Q_{m}(\alpha(f-y))+\beta w Q_{m}(\alpha(f+y \bar{r} / r)),
\end{aligned}
$$


since $Q_{m}(x)=1$ for $x \geq 1$. It remains to prove (8) for

$$
0 \leq(\bar{\alpha} / \alpha)(r / \bar{r}) f<y<B(f)
$$

Since (9) implies that

$$
0<\alpha(f-y)<f<\alpha(f+y \bar{r} / r)<1,
$$

it suffices to consider the following four cases separately.

Case (i): $\alpha(f-y)<f<\alpha(f+y \bar{r} / r) \leq r / \alpha$. In this case $B(f)=f$. By (6) and the induction hypothesis,

$$
\begin{aligned}
Q_{m+1}(f) & =\beta w Q_{m}(f \alpha / r) \\
& \geq \beta w\left\{\beta \bar{w} Q_{m-1}(\alpha(f \alpha / r-y \alpha / r))+\beta w Q_{m-1}(\alpha(f \alpha / r+(y \alpha / r) \bar{r} / r))\right\} \\
& =\beta \bar{w}\left\{\beta w Q_{m-1}(\alpha(f-y) \alpha / r)\right\}+\beta w\left\{\beta w Q_{m-1}(\alpha(f+y \bar{r} / r) \alpha / r)\right\} \\
& =\beta \bar{w} Q_{m}(\alpha(f-y))+\beta w Q_{m}(\alpha(f+y \bar{r} / r)),
\end{aligned}
$$

proving (8).

Case (ii): $r / \alpha \leq \alpha(f-y)<f<\alpha(f+y \bar{r} / r)$. Let $f^{\prime}=(f-r / \alpha) /(\bar{r} / \alpha)$ and $y^{\prime}=\left(y+\bar{\alpha} \alpha^{-2} r\right) \alpha / \bar{r}$. Note that $0<y^{\prime} \leq f^{\prime}<1$ (since $r / \alpha \leq \alpha(f-y)$ implies that $\left.y^{\prime} \leq f^{\prime}\right)$ and that $\alpha(f-B(f))=f^{\prime}$. By (7) and the induction hypothesis,

$$
\begin{aligned}
Q_{m+1}(f)= & \beta \bar{w} Q_{m}\left(f^{\prime}\right)+\beta w \\
\geq & \beta \bar{w}\left\{\beta \bar{w} Q_{m-1}\left(\alpha\left(f^{\prime}-y^{\prime}\right)\right)+\beta w Q_{m-1}\left(\alpha\left(f^{\prime}+y^{\prime} \frac{\bar{r}}{r}\right)\right)\right\}+\beta w \\
\geq & \beta \bar{w}\left\{\beta \bar{w} Q_{m-1}\left(\alpha\left(f^{\prime}-y^{\prime}\right)\right)+\beta w\right\} \\
& +\beta w\left\{\beta \bar{w} Q_{m-1}\left(\frac{\alpha(f+y \bar{r} / r)-r / \alpha}{\bar{r} / \alpha}\right)+\beta w\right\} \\
= & \beta \bar{w} Q_{m}(\alpha(f-y))+\beta w Q_{m}\left(\alpha\left(f+y \frac{\bar{r}}{r}\right)\right),
\end{aligned}
$$

proving (8), where the second inequality follows from the fact that

$$
\alpha\left(f^{\prime}+y^{\prime} \frac{\bar{r}}{r}\right)-\frac{\alpha(f+y \bar{r} / r)-r / \alpha}{\bar{r} / \alpha}=\frac{\bar{\alpha}}{\bar{r}} \geq 0
$$

and the last equality follows from (7) and the fact that

$$
\alpha\left(f^{\prime}-y^{\prime}\right)=\frac{\alpha(f-y)-r / \alpha}{\bar{r} / \alpha} .
$$

Case (iii): $\alpha(f-y)<f<r / \alpha<\alpha(f+y \bar{r} / r)$. Since $y<f=B(f)$, we have

$$
r / \alpha<\alpha(f+y \bar{r} / r)<f \alpha / r<1 .
$$


Thus, by (6) and (7),

$$
\begin{aligned}
Q_{m+1}(f) & =\beta w Q_{m}(f \alpha / r) \\
& =\beta w\left\{\beta \bar{w} Q_{m-1}\left(\frac{f \alpha / r-r / \alpha}{\bar{r} / \alpha}\right)+\beta w\right\} \\
& =\beta \bar{w}\left\{\beta w Q_{m-1}\left(\left(f \alpha / \bar{r}-\alpha^{-1} r^{2} / \bar{r}\right) \alpha / r\right)\right\}+\beta^{2} w^{2} \\
& =\beta \bar{w} Q_{m}\left(f \alpha / \bar{r}-\alpha^{-1} r^{2} / \bar{r}\right)+\beta^{2} w^{2} \\
Q_{m}(\alpha(f-y)) & =\beta w Q_{m-1}\left((f-y) \alpha^{2} / r\right), \\
Q_{m}\left(\alpha\left(f+y \frac{\bar{r}}{r}\right)\right) & =\beta \bar{w} Q_{m-1}\left(\frac{\alpha(f+y \bar{r} / r)-r / \alpha}{\bar{r} / \alpha}\right)+\beta w .
\end{aligned}
$$

It follows that (8) is equivalent to

$$
Q_{m}\left(f \frac{\alpha}{\bar{r}}-\alpha^{-1} \frac{r^{2}}{\bar{r}}\right) \geq \beta w Q_{m-1}\left((f-y) \frac{\alpha^{2}}{r}\right)+\beta w Q_{m-1}\left(\frac{\alpha(f+y \bar{r} / r)-r / \alpha}{\bar{r} / \alpha}\right) .
$$

(Note that here $f \alpha / \bar{r}-\alpha^{-1} r^{2} / \bar{r},(f-y) \alpha^{2} / r$, and $[\alpha(f+y \bar{r} / r)-r / \alpha] /(\bar{r} / \alpha)$, the various arguments of $Q_{m}$ and $Q_{m-1}$, all lie in the set $[0,1)$.)

Since $w \leq \frac{1}{2}$, the monotonicity of $Q_{n}(x)$ in $x$ and $n$ implies (11) if

$$
f \frac{\alpha}{\bar{r}}-\alpha^{-1} \frac{r^{2}}{\bar{r}} \geq \max \left\{(f-y) \frac{\alpha^{2}}{r}, \frac{\alpha(f+y \bar{r} / r)-r / \alpha}{\bar{r} / \alpha}\right\} .
$$

It therefore suffices to consider the following two subcases.

Subcase (iii.1):

$$
f \frac{\alpha}{\bar{r}}-\alpha^{-1} \frac{r^{2}}{\bar{r}}<\max \left\{(f-y) \frac{\alpha^{2}}{r}, \frac{\alpha(f+y \bar{r} / r)-r / \alpha}{\bar{r} / \alpha}\right\}=(f-y) \frac{\alpha^{2}}{r} .
$$

Let $f^{\prime} \equiv f^{\prime}(f, y)$ and $y^{\prime} \equiv y^{\prime}(f, y)$ be as defined in Lemma 4, meaning that $0 \leq y^{\prime} \leq f^{\prime}$. Since $r \geq \frac{1}{2}$ and $\alpha(f+y \bar{r} / r)>r / \alpha$, we have

$$
f \alpha / \bar{r}-\alpha^{-1} r^{2} / \bar{r}-f^{\prime}=(r / \bar{r}-1)[\alpha(f+y \bar{r} / r)-r / \alpha] \geq 0 .
$$

It follows from Lemma 4 , the induction hypothesis, and $w \leq \frac{1}{2}$ that

$$
\begin{aligned}
Q_{m}\left(f \frac{\alpha}{\bar{r}}-\alpha^{-1} \frac{r^{2}}{\bar{r}}\right) & \geq Q_{m}\left(f^{\prime}\right) \\
& \geq \beta \bar{w} Q_{m-1}\left(\alpha\left(f^{\prime}-y^{\prime}\right)\right)+\beta w Q_{m-1}\left(\alpha\left(f^{\prime}+y^{\prime} \frac{\bar{r}}{r}\right)\right) \\
& \geq \beta w Q_{m-1}\left(\frac{\alpha(f+y \bar{r} / r)-r / \alpha}{\bar{r} / \alpha}\right)+\beta w Q_{m-1}\left((f-y) \frac{\alpha^{2}}{r}\right),
\end{aligned}
$$

proving (11) and, hence, (8).

Subcase (iii.2):

$$
f \frac{\alpha}{\bar{r}}-\alpha^{-1} \frac{r^{2}}{\bar{r}}<\max \left\{(f-y) \frac{\alpha^{2}}{r}, \frac{\alpha(f+y \bar{r} / r)-r / \alpha}{\bar{r} / \alpha}\right\}=\frac{\alpha(f+y \bar{r} / r)-r / \alpha}{\bar{r} / \alpha} .
$$


Let $f^{\prime} \equiv f^{\prime}(f, y)$ and $y^{\prime} \equiv y^{\prime}(f, y)$ be as defined in Lemma 5, meaning that $0 \leq y^{\prime} \leq f^{\prime}$. Since $r \geq \frac{1}{2}$ and $f>y$, we have

$$
f \frac{\alpha}{\bar{r}}-\alpha^{-1} \frac{r^{2}}{\bar{r}}-f^{\prime}=\frac{(r-\bar{r})(f-y)}{r / \alpha} \geq 0 .
$$

By Lemma 5, the induction hypothesis, and $w \leq \frac{1}{2}$,

$$
\begin{aligned}
Q_{m}\left(f \frac{\alpha}{\bar{r}}-\alpha^{-1} \frac{r^{2}}{\bar{r}}\right) & \geq Q_{m}\left(f^{\prime}\right) \\
& \geq \beta \bar{w} Q_{m-1}\left(\alpha\left(f^{\prime}-y^{\prime}\right)\right)+\beta w Q_{m-1}\left(\alpha\left(f^{\prime}+y^{\prime} \frac{\bar{r}}{r}\right)\right) \\
& \geq \beta w Q_{m-1}\left((f-y) \frac{\alpha^{2}}{r}\right)+\beta w Q_{m-1}\left(\frac{\alpha(f+y \bar{r} / r)-r / \alpha}{\bar{r} / \alpha}\right),
\end{aligned}
$$

proving (11) and, hence, (8).

Case (iv): $\alpha(f-y)<r / \alpha \leq f<\alpha(f+y \bar{r} / r)$. By (9) and (2), $y<B(f)=\left(\alpha^{-1}-f\right) r / \bar{r}$, so

$$
\frac{r}{\alpha}>\alpha(f-y)>\alpha(f-B(f))=\frac{f-r / \alpha}{\bar{r} / \alpha} .
$$

Then, by (6) and (7),

$$
\begin{aligned}
Q_{m+1}(f) & =\beta \bar{w} Q_{m}\left(\frac{f-r / \alpha}{\bar{r} / \alpha}\right)+\beta w \\
& =\beta^{2} \bar{w} w Q_{m-1}\left(\frac{f-r / \alpha}{\bar{r} / \alpha} \frac{\alpha}{r}\right)+\beta w, \\
Q_{m}(\alpha(f-y)) & =\beta w Q_{m-1}\left((f-y) \alpha^{2} / r\right), \\
Q_{m}\left(\alpha\left(f+y \frac{\bar{r}}{r}\right)\right) & =\beta \bar{w} Q_{m-1}\left(\frac{\alpha(f+y \bar{r} / r)-r / \alpha}{\bar{r} / \alpha}\right)+\beta w .
\end{aligned}
$$

It follows that (8) is equivalent to

$$
\begin{aligned}
\bar{\beta}+ & \beta \bar{w}+\beta \bar{w} Q_{m-1}\left(\frac{(f-r / \alpha) \alpha / r}{\bar{r} / \alpha}\right) \\
& \geq \beta \bar{w} Q_{m-1}\left((f-y) \frac{\alpha^{2}}{r}\right)+\beta \bar{w} Q_{m-1}\left(\frac{\alpha(f+y \bar{r} / r)-r / \alpha}{\bar{r} / \alpha}\right) .
\end{aligned}
$$

(Note that $[(f-r / \alpha) \alpha / r] /(\bar{r} / \alpha),(f-y) \alpha^{2} / r$ and $[\alpha(f+y \bar{r} / r)-r / \alpha] /(\bar{r} / \alpha)$, the various arguments of $Q_{m-1}$, all lie in the set $[0,1)$.)

Inequality (12) holds trivially if

$$
\frac{(f-r / \alpha) \alpha / r}{\bar{r} / \alpha} \geq(f-y) \frac{\alpha^{2}}{r} \quad \text { or } \quad \frac{(f-r / \alpha) \alpha / r}{\bar{r} / \alpha} \geq \frac{\alpha(f+y \bar{r} / r)-r / \alpha}{\bar{r} / \alpha},
$$

i.e. if

$$
y \geq\left(\alpha^{-1}-f\right) r / \bar{r} \quad \text { or } \quad y \leq f-r / \alpha+r^{2} \bar{\alpha} /\left(\bar{r} \alpha^{2}\right) .
$$


If $\left(\alpha^{-1}-f\right) r / \bar{r} \leq f-r / \alpha+r^{2} \bar{\alpha} /\left(\bar{r} \alpha^{2}\right)$ (i.e. $\left.f \geq 2 r / \alpha-r^{2} / \alpha^{2}\right)$, then we have finished. Thus, it remains to prove (12) when

$$
f<2 r / \alpha-r^{2} / \alpha^{2}
$$

and

$$
f \frac{r \bar{\alpha}}{\bar{r} \alpha} \leq f-\frac{r}{\alpha}+\frac{r^{2} \bar{\alpha}}{\bar{r} \alpha^{2}}<y<\left(\alpha^{-1}-f\right) \frac{r}{\bar{r}}=B(f)
$$

Note that the left-most inequality in (14) follows since $r / \alpha \leq f<1$ implies that $r \bar{\alpha}<\bar{r} \alpha$ and

$$
f-\frac{r}{\alpha}+\frac{r^{2} \bar{\alpha}}{\bar{r} \alpha^{2}}=f-\frac{r}{\alpha}\left(1-\frac{r \bar{\alpha}}{\bar{r} \alpha}\right) \geq f-f\left(1-\frac{r \bar{\alpha}}{\bar{r} \alpha}\right)=f \frac{r \bar{\alpha}}{\bar{r} \alpha} .
$$

Observing that $r / \alpha \leq f \alpha / r-1+r / \alpha<1$ (by (13)), we have

$$
\begin{aligned}
Q_{m}\left(f \frac{\alpha}{r}-1+\frac{r}{\alpha}\right) & =\beta \bar{w} Q_{m-1}\left(\frac{f \alpha / r-1+r / \alpha-r / \alpha}{\bar{r} / \alpha}\right)+\beta w \\
& =\beta \bar{w} Q_{m-1}\left(\frac{(f-r / \alpha) \alpha / r}{\bar{r} / \alpha}\right)+\beta w,
\end{aligned}
$$

which implies that (12) is equivalent to

$$
\begin{aligned}
\bar{\beta}+ & \beta(1-2 w)+Q_{m}\left(f \frac{\alpha}{r}-1+\frac{r}{\alpha}\right) \\
& \geq \beta \bar{w} Q_{m-1}\left((f-y) \frac{\alpha^{2}}{r}\right)+\beta \bar{w} Q_{m-1}\left(\frac{\alpha(f+y \bar{r} / r)-r / \alpha}{\bar{r} / \alpha}\right) .
\end{aligned}
$$

Since $w \leq \frac{1}{2}$ and $\bar{\beta}+\beta(1-2 w)+1 \geq 2 \beta \bar{w},(15)$ follows from the monotonicity of $Q_{n}(x)$ in $x$ and $n$ if

$$
f \frac{\alpha}{r}-1+\frac{r}{\alpha} \geq \max \left\{(f-y) \frac{\alpha^{2}}{r}, \frac{\alpha(f+y \bar{r} / r)-r / \alpha}{\bar{r} / \alpha}\right\} .
$$

It remains to prove (15) for the following two subcases.

Subcase (iv.1):

$$
f \frac{\alpha}{r}-1+\frac{r}{\alpha}<\max \left\{(f-y) \frac{\alpha^{2}}{r}, \frac{\alpha(f+y \bar{r} / r)-r / \alpha}{\bar{r} / \alpha}\right\}=(f-y) \frac{\alpha^{2}}{r}
$$

Let $f^{\prime} \equiv f^{\prime}(f, y)$ and $y^{\prime} \equiv y^{\prime}(f, y)$ be as defined in Lemma 4, meaning that $0 \leq y^{\prime} \leq f^{\prime}$. Since $r \geq \frac{1}{2}$ and $\alpha(f-y)<r / \alpha$, we have

$$
f \frac{\alpha}{r}-1+\frac{r}{\alpha}-f^{\prime}=\frac{1-2 r}{r} \alpha(f-y)-1+2 \frac{r}{\alpha} \geq \frac{1-2 r}{r} \frac{r}{\alpha}-1+\frac{2 r}{\alpha}=\frac{1}{\alpha}-1 \geq 0 .
$$


By Lemma 4 and the induction hypothesis,

$$
\begin{aligned}
& Q_{m}\left(f \frac{\alpha}{r}-1+\frac{r}{\alpha}\right) \\
& \quad \geq Q_{m}\left(f^{\prime}\right) \\
& \quad \geq \beta \bar{w} Q_{m-1}\left(\alpha\left(f^{\prime}-y^{\prime}\right)\right)+\beta w Q_{m-1}\left(\alpha\left(f^{\prime}+y^{\prime} \frac{\bar{r}}{r}\right)\right) \\
& \quad=\beta \bar{w} Q_{m-1}\left(\frac{\alpha(f+y \bar{r} / r)-r / \alpha}{\bar{r} / \alpha}\right)+\beta w Q_{m-1}\left((f-y) \frac{\alpha^{2}}{r}\right) \\
& \quad \geq \beta \bar{w} Q_{m-1}\left(\frac{\alpha(f+y \bar{r} / r)-r / \alpha}{\bar{r} / \alpha}\right)+\beta \bar{w} Q_{m-1}\left((f-y) \frac{\alpha^{2}}{r}\right)-\beta(1-2 w),
\end{aligned}
$$

from which (15) (and, hence, (12) and (8)) follows.

Subcase (iv.2):

$$
f \frac{\alpha}{r}-1+\frac{r}{\alpha}<\max \left\{(f-y) \frac{\alpha^{2}}{r}, \frac{\alpha(f+y \bar{r} / r)-r / \alpha}{\bar{r} / \alpha}\right\}=\frac{\alpha(f+y \bar{r} / r)-r / \alpha}{\bar{r} / \alpha} .
$$

Let $f^{\prime} \equiv f^{\prime}(f, y)$ and $y^{\prime} \equiv y^{\prime}(f, y)$ be as defined in Lemma 5, meaning that $0 \leq y^{\prime} \leq f^{\prime}$. Since $r \geq \frac{1}{2}$ and $\alpha(f+y \bar{r} / r)<1$ (by (10)), we have

$$
f \frac{\alpha}{r}-1+\frac{r}{\alpha}-f^{\prime}=\left(1-\frac{r}{\bar{r}}\right) \alpha\left(f+\frac{y \bar{r}}{r}\right)-1+\frac{r}{\bar{r} \alpha} \geq 1-\frac{r}{\bar{r}}-1+\frac{r}{\bar{r} \alpha}=\frac{r \bar{\alpha}}{\bar{r} \alpha} \geq 0 .
$$

By Lemma 5 and the induction hypothesis,

$$
\begin{aligned}
& Q_{m}\left(f \frac{\alpha}{r}-1+\frac{r}{\alpha}\right) \\
& \quad \geq Q_{m}\left(f^{\prime}\right) \\
& \quad \geq \beta \bar{w} Q_{m-1}\left(\alpha\left(f^{\prime}-y^{\prime}\right)\right)+\beta w Q_{m-1}\left(\alpha\left(f^{\prime}+y^{\prime} \frac{\bar{r}}{r}\right)\right) \\
& \quad=\beta \bar{w} Q_{m-1}\left((f-y) \frac{\alpha^{2}}{r}\right)+\beta w Q_{m-1}\left(\frac{\alpha(f+y \bar{r} / r)-r / \alpha}{\bar{r} / \alpha}\right) \\
& \quad \geq \beta \bar{w} Q_{m-1}\left((f-y) \frac{\alpha^{2}}{r}\right)+\beta \bar{w} Q_{m-1}\left(\frac{\alpha(f+y \bar{r} / r)-r / \alpha}{\bar{r} / \alpha}\right)-\beta(1-2 w),
\end{aligned}
$$

from which (15) (and, hence, (12) and (8)) follows.

This completes the proof.

\section{References}

[1] Breiman, L. (1961). Optimal gambling systems for favorable games. In Proc. 4th Berkeley Symp. Math. Statist. Prob., Vol. 1, University of California Press, Berkeley, CA, pp. 65-78.

[2] Chen, R. (1976). Subfair discounted red-and-black game with a house limit. J. Appl. Prob. 13, 608-613.

[3] Chen, R. (1977). Subfair primitive casino with a discount factor. Z. Wahrscheinlichkeitsth. 39, 167-174.

[4] Chen, R. (1978). Subfair 'red-and-black' in the presence of inflation. Z. Wahrscheinlichkeitsth. 42, $293-301$.

[5] Chen, R. and Zame, A. (1979). On discounted subfair primitive casino. Z. Wahrscheinlichkeitsth. 49, $257-266$. 
[6] Chen, R. W., Shepp, L. A. And Zame, A. (2004). A bold strategy is not always optimal in the presence of inflation. J. Appl. Prob. 41, 587-592.

[7] Chen, R. W., Shepp, L. A., Yao, Y.-C. and Zhang C.-H. (2005). On optimality of bold play for primitive casinos in the presence of inflation. J. Appl. Prob. 42, 121-137.

[8] Dubins, L. E. (1968). A simpler proof of Smith's roulette theorem. Ann. Math. Statist. 39, 390-393.

[9] Dubins, L. E. (1998). Discrete red-and-black with fortune-dependent win probabilities. Prob. Eng. Inf. Sci. 12, 417-424.

[10] Dubins, L. E. And Savage, L. J. (1965). How to Gamble if You Must. Inequalities for Stochastic Processes. McGraw-Hill, New York.

[11] Dubins, L. E. And Savage, L. J. (1976). Inequalities for Stochastic Processes. How to Gamble if You Must. Corrected republication of the 1965 edition. Dover, New York.

[12] Heath, D. C. and Sudderth, W. D. (1974). Continuous-time gambling problems. Adv. Appl. Prob. 6, 651-665.

[13] Heath, D. C., Pruitt, W. E. and Sudderth, W. D. (1972). Subfair red-and-black with a limit. Proc. Amer. Math. Soc. 35, 555-560.

[14] Klugman, S. (1977). Discounted and rapid subfair red-and-black. Ann. Statist. 5, 734-745.

[15] Kulldorff, M. (1993). Optimal control of favorable games with a time limit. SIAM J. Control Optimization 31, 52-69.

[16] Maitra, A. P. And Sudderth, W. D. (1996). Discrete Gambling and Stochastic Games (Appl. Math. (New York) 32). Springer, New York.

[17] Pestien, V. And Sudderth, W. D. (1985). Continuous-time red-and-black: how to control a diffusion to a goal. Math. Operat. Res. 10, 599-611.

[18] Pestien, V. and Sudderth, W. D. (1988). Continuous-time casino problems. Math. Operat. Res. 13, $364-376$.

[19] Ross, S. M. (1974). Dynamic programming and gambling models. Adv. Appl. Prob. 6, 593-606.

[20] Schweinsberg, J. (2005). Improving on bold play when the gambler is restricted. J. Appl. Prob. 42, 321-333.

[21] Secchi, P. (1997). Two-person red-and-black stochastic games. J. Appl. Prob. 34, 107-126.

[22] Sмiтh, G. J. (1967). Optimal strategy at roulette. Z. Wahrscheinlichkeitsth. 8, 91-100.

[23] Sudderth, W. D. and Weerasinghe, A. (1989). Controlling a process to a goal in finite time. Math. Operat. Res. 14, 400-409.

[24] Wilkins, J. E. (1972). The bold strategy in presence of house limit. Proc. Amer. Math. Soc. 32, 567-570. 